\title{
Optimization of Textured Surface in 2D Parallel Bearings Governed by the Reynolds Equation Including Cavitation and Temperature
}

\author{
Agata Guzek*, Pawel Podsiadlo and Gwidon W. Stachowiak \\ Tribology Laboratory, School of Mechanical and Chemical Engineering, The University of Western Australia \\ 35 Stirling Hwy, Crawley, Western Australia 6009 \\ *Corresponding author: agata@mech.uwa.edu.au
}

\begin{abstract}
( Manuscript received 28 December 2011; accepted 14 September 2012; published 31 January 2013 )
( Presented at Symposium S2: Multi-Scale Surface Texturing in Tribology in the International Tribology Conference Hiroshima 2011)
\end{abstract}

\begin{abstract}
One-dimensional textured parallel bearings have been successfully optimized for the maximum load capacity or the minimum friction coefficient using a unified computational approach. However, there is no efficient approach allowing for the optimization of two-dimensional (2D) bearings. The work conducted is, in most cases, by "trial and error", i.e. changes are introduced and their effects studied, either experimentally or through numerical parametric studies. This is time consuming and costly. In this paper, a uniform approach to the optimization of surface textures in 2D bearings, based on nonlinear programming routines, is proposed. The approach aims at finding the optimal textured surfaces that support the maximum load and/or minimize friction coefficient. Examples of parallel hydrodynamic bearings with surfaces textured by rectangular or elliptical dimples and governed by Reynolds equations, considering mass-conserving cavitation and decrease in viscosity due to temperature change are optimized. Results of the optimization are comparable to those obtained using an exhaustive search and found in literature.
\end{abstract}

Keywords: hydrodynamic bearings, surface texture, geometric shapes, shape optimization

\section{Introduction}

Surface texturing can improve tribological characteristics of mechanical components [1-3]. Surface textures in different forms have been investigated for their possible reduction of friction and wear, increase of load-carrying capacity and avoidance of seizure and damage of contact surfaces. The research question is to find textured surfaces that would maximize the load carrying capacity or minimize the friction coefficient. One-dimensional (1D) optimal textured surfaces were already established either analytically [4] or through numerical parametric search [5,6]. A unified computational approach that allows finding optimal 1D textured surfaces for a wide range of hydrodynamic bearings governed by the Reynolds equation, accounting for non-Newtonian lubrication and temperature effects was developed previously by the authors of this work and presented in [7].

One of the main problems that still remain unsolved is finding an optimal textured surface for two-dimensional (2D) bearings. Various approaches have been used so far for this optimization, including analytical solution of governing equations, experiments and numerical methods.

Analytical solutions for optimal shapes were obtained, e.g. with the use of the calculus of variations [4,8-10]. However, analytical solution has been possible only for basic $2 \mathrm{D}$ cases due to complexity of governing equations. As a result of this limitation, other optimization approaches were developed, such as experimental search. To perform the search, it is necessary to manufacture bearings with various textured surfaces and conduct experiments, measuring tribological characteristics of mechanical components with different surface texture parameters. Extensive experimental studies of laser surface textured mechanical components were performed by Etsion et al. [3,11-13]. However, experimental search is time-consuming and the cost of performing many tests is often prohibitively high.

Recently, with the growth of computing power available, there has been an increased interest in using numerical methods for textured surface optimization. In many studies, optimization was performed through parametric numerical search. Numerical simulations were used to obtain pressure fields from the Reynolds equation, load capacity and friction for parallel thrust bearings textured with spherical dimples of different radii and area ratios [12]. Recently, an analysis of a bearing governed by the 2D Reynolds equation including mass-conserving cavitation was made by Dobrica et al. [14]. A parametric theoretical and experimental study of 
optimal elliptical dimples was also performed by Ma et al. [15]. 2D fluid flow in a journal bearing was also studied by Cupillard et al. using the Navier-Stokes equations, where load capacity and friction were calculated for varying dimple depths and eccentricity ratios [16], including a thermohydrodynamic analysis of 3D textured sliders [17]. However, the approach based on numerical exhaustive search is time-consuming and costly in terms of computing power.

For arbitrary texture profiles, shape optimization problem has been solved using unconstrained and constrained optimization methods. In [18], unconstrained nonlinear programming lattice search and simplex methods were used. A 2D lubrication model based on the Reynolds equation was analysed to obtain the optimal film profiles for elliptical and slider bearings. Recently, van Ostayen conducted partial differential equation (PDE) constrained optimization of a parallel slider profile applying the adjoint approach $[19,20]$. In his studies, bearing profile was treated as a mathematical function permitted to take arbitrary values and its optimal shape was calculated. Some attempts at textured surface optimization were also made with the use of heuristic algorithms such as genetic algorithms [21]. Size and depth of the microdimples were optimized with the aim of minimizing the friction. However, heuristic algorithms are often criticized as they do not guarantee to find the optimum or even a satisfactory near-optimal solution [22].

A uniform computational approach for optimizing parameters of a surface textured with dimples has not been established so far.

This study aims at developing an approach for finding optimal surfaces textured by rectangular or elliptical dimples. Optimal texture area density and height ratio are sought. Also, optimal length of the textured portion of the slider is determined. The optimization is performed with load capacity and friction coefficient taken as objective functions. Investigated bearings are governed by the 2D Reynolds equation for a wide range of cases, taking into account phenomena such as mass-conserving cavitation and temperature effects in bearings.

Previous studies showed that cavitation occurs in bearings with textured surfaces [12]. Because the simple and commonly used Reynolds model was shown to be inaccurate, underestimating the cavitated area [23], employment of a mass-conserving approach, following the Jakobsson-Floberg-Olsson (JFO) cavitation theory [24], is preferred. Such an algorithm was first developed by Elrod $[25,26]$, which was further extended by Ausas [23]. This version of the algorithm is applied in our current work. The loss of viscosity due to heating may cause significant reduction in bearing load capacity [27]. To include thermal effects in a bearing, an equation describing temperature changes - the energy equation is solved simultaneously with the Reynolds equation.

\section{Method description}

\subsection{Textured surface optimization (TSO)}

The optimization of textured surface in 2D bearings can be stated as a nonlinearly constrained optimization problem in the following way:

Find the textured surface that maximizes (minimizes) a load capacity (friction coefficient)

$$
g(h(x, y))
$$

Subject to constraints:

- The Reynolds equation $R E(p(x, y), h(x, y))$ is satisfied

- The boundary conditions that pressure vanishes at bearing edges, i.e. $p(0, y)=p(L, y)=0$ and $p(x, 0)=p(x, B)=0$

where $g$ is the objective function that represents a load $W=\int_{0}^{L} \int_{0}^{B} p(x, y) d x d y$ or a friction coefficient, $f=\frac{F}{W}$ where $F=\int_{0}^{L} \int_{0}^{B} \tau(x, y) d x d y$ is the friction force

\subsection{Bearing and texture geometry}

A schematic diagram of a square parallel slider bearing is shown in Fig. 1(a). The lower surface (the

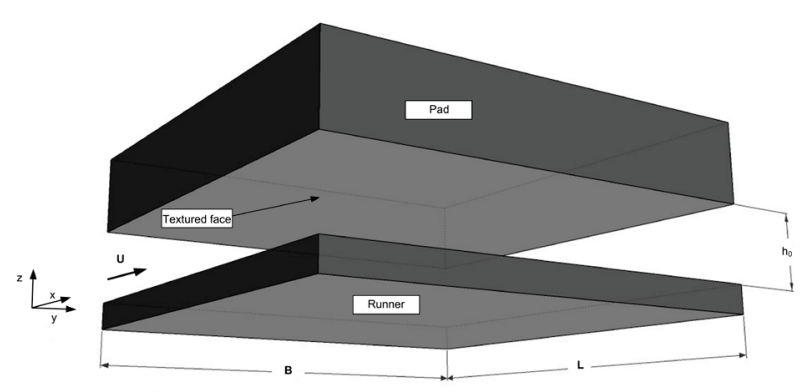

(a)

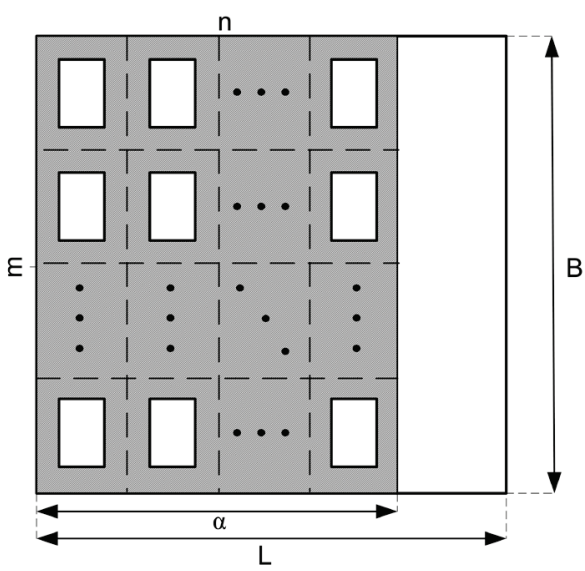

(b)

Fig. 1 Schematic diagrams of (a) the parallel slider bearing and (b) the textured face of the pad 
runner) slides at a constant velocity $\boldsymbol{U}$ and the upper surface (the pad) is textured and fixed. The minimum film thickness between the surfaces is $h_{0}$. The pad is textured with either rectangular or elliptical dimples. An example of the pad that has $m \times n$ rectangular dimple cells is shown in Fig. 1(b).

\subsubsection{Rectangular dimples}

For rectangular dimples the film height within cell is defined as (Fig. 2):

$$
h\left(X_{r}, Y_{r}\right)=\left\{\begin{array}{l}
h_{D} \text { for }\left(\left|X_{r}\right| \leq r_{1}\right) \cap\left(\left|Y_{r}\right| \leq r_{2}\right) \\
h_{0} \text { elsewhere }
\end{array}\right.
$$

\subsubsection{Elliptical dimples}

For elliptical dimples the film height within cell is defined as (Fig. 3):

$$
h\left(X_{r}, Y_{r}\right)=\left\{\begin{array}{l}
h_{0}+\mathrm{e} \sqrt{r_{p}^{2}-X_{r}^{2}-Y_{r}^{2}} \text { for } X_{r}^{2}+Y_{r}^{2} \leq r_{p}^{2} \\
h_{0} \quad \text { elsewhere }
\end{array}\right.
$$

\subsection{Optimal parameter selection}

TSO problem can be formulated as an optimal parameter selection problem, i.e. finding the maximum (minimum) load capacity (friction coefficient)

$$
g(h(x, y, \boldsymbol{a}))
$$

with respect to the parameter vector $\boldsymbol{a}=\left[H_{r} A_{r} \alpha\right]$, and subject to the constraint conditions stated in 2.1; where $H_{r}, A_{r}$ and $\alpha$ are the parameters of the textured surface, denoting height ratio, area density and textured portion of slider, respectively.

The parameters of optimization were chosen in line with previous studies [12,14,17,28-30]. In particular, Brizmer et al. [12] studied effects of the texture density and dimple depth on the hydrodynamic performance of a parallel thrust bearing. The effects of the texture density was also investigated in [17] for a pad slider. Other studies show that the size of textured fraction of the component is important, because only partial texturing generates the hydrodynamic lift as opposed to the full texturing in journal bearings, parallel sliders and mechanical seals [14,28-30].

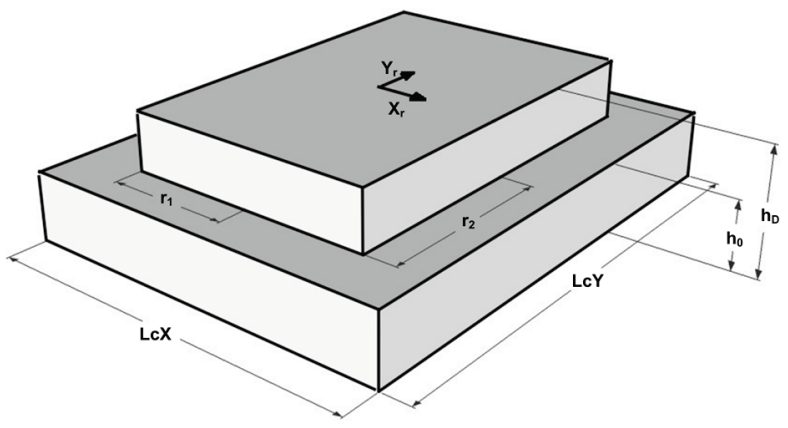

Fig. 2 Rectangular dimple cell

\subsection{Nonlinear programming}

The TSO problem can be formulated as a nonlinear mathematical programming problem (NLMP), i.e.

Minimize (or maximize) an objective functional

$$
\min _{\boldsymbol{a}} g(\boldsymbol{a})
$$

subject to box constraints: $\boldsymbol{a}_{\boldsymbol{l b}} \leq \boldsymbol{a} \leq \boldsymbol{a}_{\boldsymbol{u} \boldsymbol{b}}$,

where $\boldsymbol{a}_{l b}$ and $\boldsymbol{a}_{u b}$ are the vectors of lower and upper bounds on parameters.

\subsection{Numerical method}

The NLMP is solved as a sequence of quadratic programming (SQP) subproblems [31]. Each QP subproblem is defined as

$$
\min _{\boldsymbol{d}} \frac{1}{2} \boldsymbol{d}^{T} \boldsymbol{H}_{k} \boldsymbol{d}+\nabla g\left(\boldsymbol{a}_{k}\right)^{T} \boldsymbol{d}
$$

subject to: $\boldsymbol{a}_{\boldsymbol{l b}} \leq \boldsymbol{a} \leq \boldsymbol{a}_{\boldsymbol{u b}}$,

where:

$\nabla g$ is the gradient of the objective functional,

$\boldsymbol{H}_{k}$ is the Hessian, a positive definite symmetric matrix,

$\boldsymbol{d}$ is the solution of the QP subproblem.

At each iteration the parameter $\boldsymbol{a}$ is updated as follows

$$
\boldsymbol{a}_{k+1}=\boldsymbol{a}_{k}+\beta_{k} \boldsymbol{d}_{k}
$$

where:

$\boldsymbol{a}_{k}, \boldsymbol{a}_{k+1}$ are values of $\boldsymbol{a}$ at subsequent iterations $k$ and $k+1$,

$\beta_{k}$ is the step length,

$\boldsymbol{d}_{k}$ is the line search direction.

The direction $\boldsymbol{d}_{\boldsymbol{k}}$ is obtained at each iteration $k$ as the solution of the QP subproblem. To determine the step length $\beta_{k}$ along the search direction $\boldsymbol{d}_{\boldsymbol{k}}$, a line search procedure is carried out. At each iteration, $\beta_{k}$ takes values of

- Unity, if it does not violate the constraints, or

- The distance along the search direction to the nearest constraint.

A positive definite quasi-Newton approximation of the Hessian $\boldsymbol{H}_{k}$ is calculated at each iteration using a Broyden-Fletcher-Goldfarb-Shanno (BFGS) method:

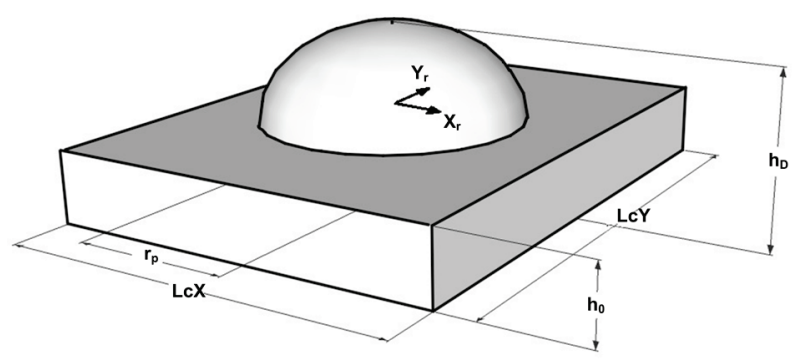

Fig. 3 Elliptical dimple cell 


$$
\boldsymbol{H}_{k+1}=\boldsymbol{H}_{k}+\frac{q_{k} q_{k}^{T}}{q_{k}^{T} \boldsymbol{s}_{k}}-\frac{\boldsymbol{H}_{k}^{T} \boldsymbol{s}_{k}^{T} \boldsymbol{s}_{k} \boldsymbol{H}_{k}}{\boldsymbol{s}_{k}^{T} \boldsymbol{H}_{k} \boldsymbol{s}_{k}}
$$

where

$$
\begin{aligned}
& \boldsymbol{s}_{k}=\boldsymbol{a}_{k+1}-\boldsymbol{a}_{k}, \\
& q_{k}=\nabla g\left(\boldsymbol{a}_{k+1}\right)-\nabla g\left(\boldsymbol{a}_{k}\right) .
\end{aligned}
$$

The solution of each QP is suboptimal and the convergence to an optimal solution is deemed when, between two subsequent iterations, either the reduction of values of $g(\boldsymbol{a})$ or the relative change in $\boldsymbol{a}$ is less than a given threshold tolerance.

Flowchart of the optimization procedure is shown in Fig. 4.

\subsection{Computational implementation}

The objective functional maximized (or minimized), i.e. the bearing load capacity (or friction coefficient), is a function of the surface texture shape parameters. In each optimization iteration, on the basis of parameters given for that iteration, the film height $h$ is generated and the Reynolds equation is solved. The value of the objective functional is then obtained. The nonlinear programming method described in the previous section seeks the maximum (or minimum) of the functional.

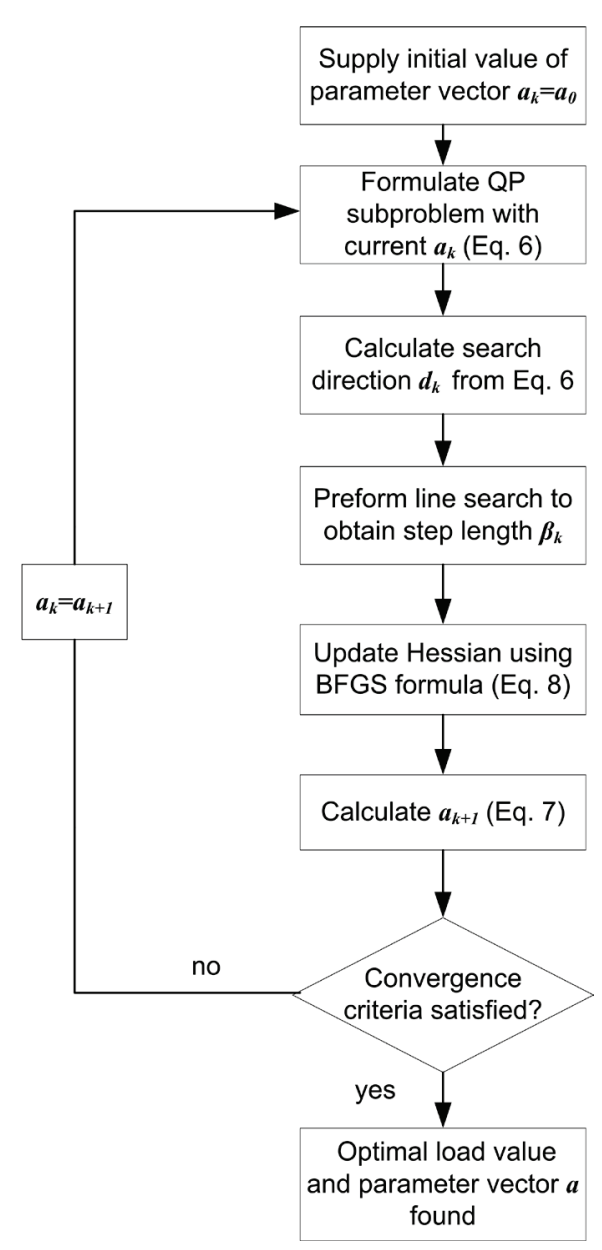

Fig. 4 Flow diagram of the optimization procedure
The computational approach applied in this work employs different methods for the Reynolds equation solution to calculate pressure distribution. For solving the equation both commercially available software, COMSOL Multiphysics [32] and specially developed finite difference and finite volume codes have been used. As COMSOL did not allow updating pressure during the solution procedure which was necessary to implement the mass-conserving algorithm, finite difference programs were developed. Additionally, in the example 4 for viscosity varying with temperature, finite volume method was used to solve the energy equation.

The use of numerical methods required choosing an appropriate computational grid size. A grid independency study was conducted to ensure that results obtained do not depend on grid size. For this study, a square $(L / B=1) 2 \mathrm{D}$ pad slider with film height $h(x, y)=$ 2.2-1.2x and five different grid sizes $(25 \times 25,50 \times 50$, $100 \times 100,200 \times 200$ and $400 \times 400)$ was used. The bearing is governed by the Reynolds equation. For each grid size the load capacity was calculated (Table 1). It was found that the grid size of $200 \times 200$ is adequate since the difference in load capacity between two consecutive grids was less than $0.01 \%$

For the grid size of $200 \times 200$ and 20 dimples in $y$ direction there are $10 \times 10$ grid points per dimple cell. This number of points is considered to be an accurate representation of the dimple geometry. Previous studies showed that using $5 \times 5$ grid points per dimple cell accurate results can be obtained [33].

Example 4 (viscosity varying with temperature) required a $3 \mathrm{D}$ grid. To capture the changes in viscosity which were as low as 0.0004 across the whole film thickness, 40 grid points in $z$ direction were used.

\section{Results}

Several examples of 2D parallel bearings textured with rectangular or elliptical dimples were selected to illustrate the optimization approach presented. Texture shapes were optimized for maximum load capacity or minimum friction coefficient. The bearings satisfy the simplifying assumptions listed in Table 2 .

The textured portion $\alpha$ is the fraction of the slider length $L$ that is covered by square dimple cells. The number of dimples in $y$ direction $(m)$ is chosen. The

Table 1 Load capacities calculated for a square pad slider using grid sizes: $25 \times 25,50 \times 50,100$ $\times 100,200 \times 200$ and $400 \times 400$

\begin{tabular}{|c|c|}
\hline Grid size & Dimensionless load capacity \\
\hline $25 \times 25$ & 0.0697765 \\
\hline $50 \times 50$ & 0.0702989 \\
\hline $100 \times 100$ & 0.0704416 \\
\hline $200 \times 200$ & 0.0704790 \\
\hline $400 \times 400$ & 0.0704821 \\
\hline
\end{tabular}


Table 2 Summary of simplifying assumptions for two-dimensional bearings

\begin{tabular}{|c|l|}
\hline Assumptions & \multicolumn{1}{c|}{ Comments } \\
\hline 1 & There is no variation of pressure across the fluid film, i.e. $\partial p / \partial z=0$. \\
\hline 2 & The lubricant flow is laminar. \\
\hline 3 & The inertial effect and the body forces are negligible. \\
\hline 4 & There is no slip at the bearing surfaces. \\
\hline 5 & There is no vertical flow across the film. \\
\hline 6 & The lubricant is incompressible and isoviscous. \\
\hline
\end{tabular}

Table 3 Optimal height ratio and dimensionless load obtained for a parallel bearing with rectangular dimples, half-Sommerfeld boundary condition

\begin{tabular}{|c|c|c|c|c|c|}
\hline \multirow{2}{*}{$m$} & \multirow{2}{*}{$n$} & \multicolumn{2}{|c|}{ Optimization } & \multicolumn{2}{c|}{ Exhaustive search } \\
\cline { 3 - 6 } & & Load & $H_{\mathrm{r}}$ & Load & $H_{\mathrm{r}}$ \\
\hline 2 & 1 & 0.0639 & 1.77 & 0.0639 & 1.77 \\
\hline 3 & 1 & 0.0519 & 1.69 & 0.0519 & 1.69 \\
\hline 5 & 3 & 0.0558 & 1.69 & 0.0558 & 1.69 \\
\hline 10 & 6 & 0.0596 & 1.70 & 0.0596 & 1.70 \\
\hline 20 & 12 & 0.0616 & 1.75 & 0.0616 & 1.75 \\
\hline
\end{tabular}

length of a single cell $L c Y$ is then calculated as $B / m$. Since the cell is square, the lengths $L c X$ and $L c Y$ are equal. When the optimal value of $\alpha$ is found, the number of dimple cells in $x$ direction is calculated as $n=$ floor $(\alpha L / L c X)$ where floor is the integer part of a number.

Example 1: Optimization of surface textured by rectangular (elliptical) dimples for the maximum load capacity

Textured surfaces with rectangular (elliptical) dimples are optimized for maximum load capacity defined as $W=\int_{0}^{L} \int_{0}^{B} p(x, y) d x d y$. The pressure in the bearing is governed by the following dimensionless 2D Reynolds equation:

$$
\frac{\partial}{\partial x^{*}}\left(h^{* 3} \frac{\partial p^{*}}{\partial x^{*}}\right)+\frac{\partial}{\partial y^{*}}\left(h^{* 3} \frac{\partial p^{*}}{\partial y^{*}}\right)=6 \frac{\partial h^{*}}{\partial x^{*}}
$$

where

$$
x^{*}=x / L, y^{*}=y / L, \quad h^{*}=h / h_{0}, p^{*}=p / p_{0}
$$

and $p_{0}$ is the reference pressure $p_{0}=U \eta L / h_{0}^{2}$.

Thus, the dimensionless load capacity is:

$$
W^{*}=\int_{0}^{L} \int_{0}^{B} p^{*} d x d y=\frac{W h_{0}^{2}}{U \eta L^{2} B}
$$

Calculations are performed for a square pad $(L / B=1)$. Cavitation is taken into account by not allowing the pressure to fall below 0 , which is equivalent to the half-Sommerfeld boundary condition. Optimal load and
Table 4 Optimal height ratio and dimensionless load obtained for a parallel bearing with rectangular dimples, Reynolds boundary condition

\begin{tabular}{|c|c|c|c|c|c|}
\hline \multirow{2}{*}{$m$} & \multirow{2}{*}{$n$} & \multicolumn{2}{|c|}{ Optimization } & \multicolumn{2}{c|}{ Exhaustive search } \\
\cline { 3 - 6 } & & Load & $H_{\mathrm{r}}$ & Load & $H_{\mathrm{r}}$ \\
\hline 2 & 1 & 0.0763 & 1.90 & 0.0763 & 1.90 \\
\hline 3 & 1 & 0.0746 & 1.74 & 0.0746 & 1.74 \\
\hline 5 & 3 & 0.0701 & 1.70 & 0.0701 & 1.70 \\
\hline 10 & 6 & 0.0626 & 1.67 & 0.0626 & 1.67 \\
\hline 20 & 12 & 0.0618 & 1.70 & 0.0618 & 1.70 \\
\hline
\end{tabular}

Table 5 Optimal height ratio and dimensionless load obtained for a parallel bearing with elliptical dimples, half-Sommerfeld boundary condition

\begin{tabular}{|c|c|c|c|c|c|}
\hline \multirow{2}{*}{$m$} & \multirow{2}{*}{$n$} & \multicolumn{2}{|c|}{ Optimization } & \multicolumn{2}{c|}{ Exhaustive search } \\
\cline { 3 - 6 } & & Load & $H_{\mathrm{r}}$ & Load & $H_{\mathrm{r}}$ \\
\hline 2 & 1 & 0.0655 & 2.21 & 0.0655 & 2.20 \\
\hline 3 & 1 & 0.0524 & 2.13 & 0.0524 & 2.13 \\
\hline 5 & 2 & 0.0568 & 2.12 & 0.0568 & 2.12 \\
\hline 10 & 5 & 0.0598 & 2.05 & 0.0598 & 2.05 \\
\hline 20 & 11 & 0.0587 & 2.14 & 0.0587 & 2.15 \\
\hline
\end{tabular}

parameter values are sought for pads with different numbers of dimples.

Height ratios $H_{\mathrm{r}}$ obtained using the optimal parameter selection approach and an exhaustive search using the half-Sommerfeld boundary condition are shown in Tables 3 and 5. The search was conducted over the range of $H_{\mathrm{r}}$ between 1.3 and 2.5 with steps of 0.01 . Results obtained from the optimization and the exhaustive search are comparable.

For rectangular dimples, the area density $A_{\mathrm{r}}$ was not optimized because the load capacity always increases with the area density. For high values of the density, denser computing meshes are required to accurately represent the dimpled area. Therefore, to avoid unnecessary numerical complexity, optimal $A_{\mathrm{r}}$ was assumed to be 0.8 . Optimal textured portion $\alpha$ was found to be approximately 0.6 for all cases. The highest value of load equal to 0.0639 was obtained for 2 dimples, with $H_{\mathrm{r}}=1.77$. Load capacity initially decreased for 3 dimples and then increased as the number of dimples became 


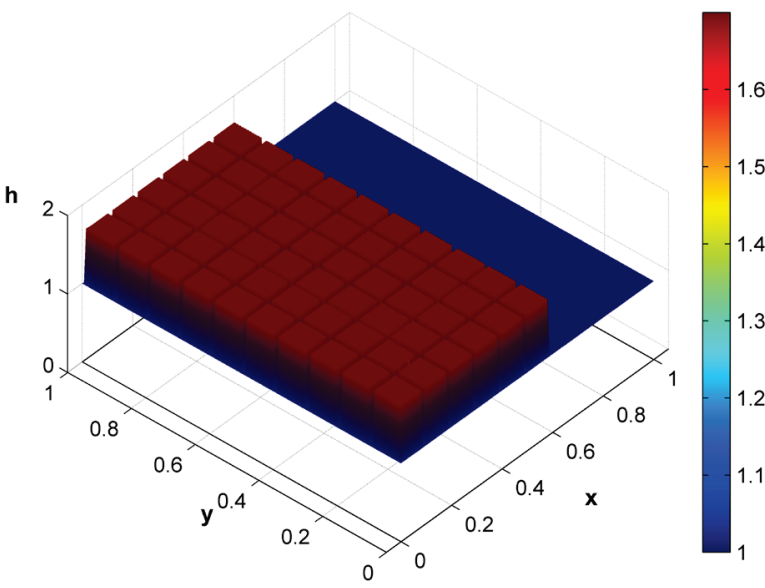

(a)

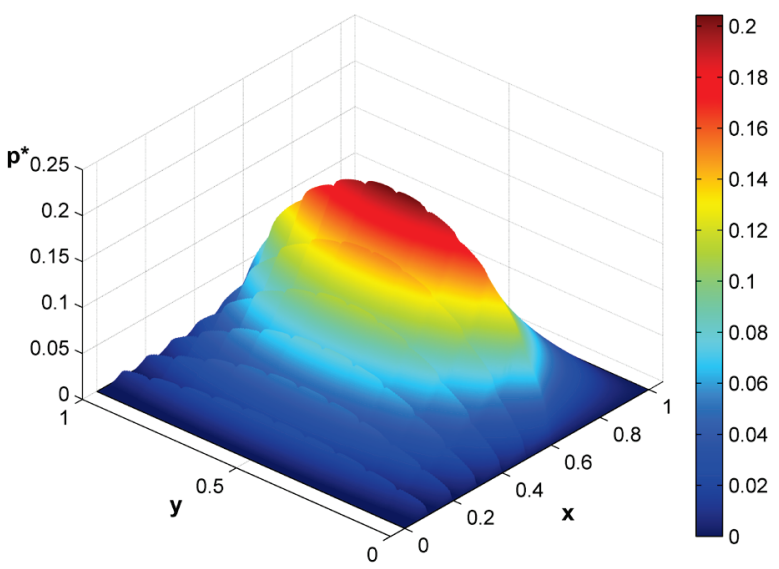

(b)

Fig. 5 Optimal film height and pressure distribution for 10 rectangular dimples in y-direction

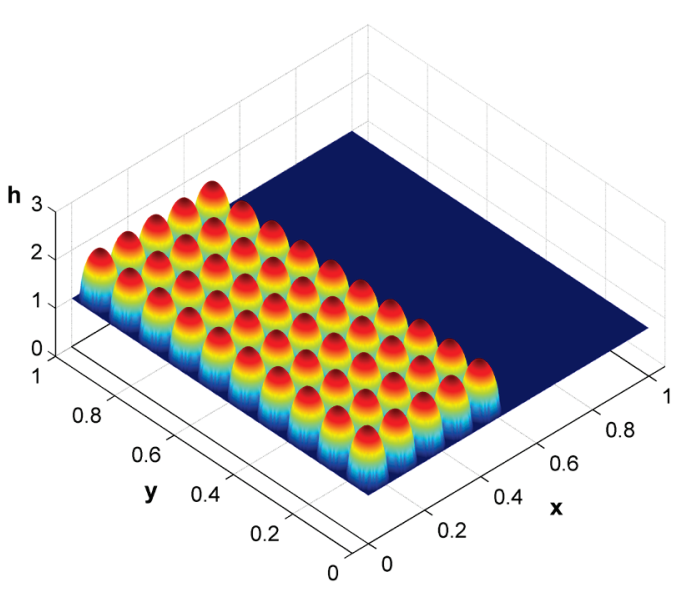

(a)

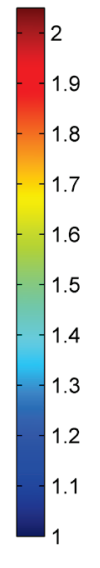

Fig. 6 Optimal film height and pressure distribution for 10 elliptical dimples in y-direction large. Results for the Reynolds boundary condition are presented in Table 4. Loads obtained with the Reynolds condition were higher.

For elliptical dimples, it was found that the load capacity increased with the area density. Thus, optimal $A_{\mathrm{r}}$ was assumed to be its maximum value, 0.78 . Optimal $\alpha$ was approximately 0.56 for all cases. The highest value of load equal to 0.0655 was obtained for 2 dimples, when $H_{\mathrm{r}}=2.21$.

Examples of optimal film heights and pressure distributions for 10 rectangular and elliptical dimples in y direction are shown in Figures 5 and 6, respectively.

Example 2: Optimization of surface textured with rectangular (elliptical) dimples for the minimum friction coefficient.

The objective functional minimized in this example is the dimensionless friction coefficient, defined as $f^{*}=\frac{F^{*}}{W^{*}}$ where $F^{*}=\int_{0}^{L} \int_{0}^{B} \tau^{*} d x d y, \tau^{*}=\frac{1}{h^{*}}+\frac{h^{*}}{2} \frac{d p^{*}}{d x}$ is the dimensionless shear stress and the pressure is governed by the Reynolds equation used in Example 1.

Optimal friction coefficient, $H_{\mathrm{r}}$ and $\alpha$ values obtained are shown in Tables 6 and 7. The exhaustive search was conducted over the range of $H_{\mathrm{r}}$ between 1.3 and 3.5 and $\alpha$ between 0.1 and 0.9 , with steps of 0.01 . Results obtained from the optimization and exhaustive search are almost identical.

For both rectangular and elliptical dimples the friction coefficient always decreased with the area density. As before the maximal assumed value of $\mathrm{A}_{\mathrm{r}}$, equal to $0.8(0.78)$ for rectangular (elliptical) dimples was used. The friction coefficients took the lowest values 
Table 6 Optimal height ratio, $\alpha$ and dimensionless friction coefficient obtained for a parallel bearing with rectangular dimples

\begin{tabular}{|c|c|c|c|c|c|c|c|}
\hline \multirow{2}{*}{$m$} & \multirow{3}{*}{$n$} & \multicolumn{3}{|c|}{ Optimization } & \multicolumn{3}{|c|}{ Exhaustive search } \\
\cline { 3 - 8 } & & $f^{*}$ & $H_{\mathrm{r}}$ & $\alpha$ & $f^{*}$ & $H_{\mathrm{r}}$ & $\alpha$ \\
\hline 2 & 1 & 13.92 & 1.80 & 0.70 & 13.92 & 1.80 & 0.84 \\
\hline 3 & 2 & 14.28 & 1.81 & 0.70 & 14.28 & 1.81 & 0.70 \\
\hline 5 & 3 & 14.58 & 1.77 & 0.65 & 14.58 & 1.76 & 0.71 \\
\hline 10 & 5 & 14.59 & 1.76 & 0.60 & 14.59 & 1.76 & 0.60 \\
\hline 20 & 11 & 14.08 & 1.81 & 0.60 & 14.08 & 1.81 & 0.61 \\
\hline
\end{tabular}

of 13.92 and 14.18 for 2 rectangular and elliptical dimples, respectively. These values are within the range from 10 to 40 for a slider textured with rectangular dimples [34] and from 5 to 50 for a step bearing [35].

Film height and pressure distribution for 20 elliptical dimples for texture parameters giving the optimal friction coefficient are shown in Fig. 7.

Example 3: Optimization of surface textured with rectangular (elliptical) dimples for the maximum load capacity, Reynolds equation considering mass-conserving cavitation

The pressure in the bearing is governed by the $2 \mathrm{D}$ Reynolds equation with the conservation of mass:

$$
\frac{\partial}{\partial x^{*}}\left(h^{*_{3}} \frac{\partial p^{*}}{\partial x^{*}}\right)+\frac{\partial}{\partial y^{*}}\left(h^{*_{3}} \frac{\partial p^{*}}{\partial y^{*}}\right)=6 \frac{\partial \theta h^{*}}{\partial x^{*}}
$$

In order to account for cavitation with the conservation of mass, assumptions of the JFO cavitation theory must hold [24]. To satisfy these assumptions, the model developed by Ausas et al. [23] was used, i.e.

$$
\begin{array}{lll}
\theta=1 & \text { if } p^{*}>0 & \text { (full film) } \\
0<\theta<1 & \text { if } p^{*}=0 & \text { (cavitated region) }
\end{array}
$$

where $\theta$ is the fractional film content [26].

Optimal results obtained for rectangular cell-centred
Table 7 Optimal height ratio, $\alpha$ and dimensionless friction coefficient obtained for a parallel bearing with elliptical dimples

\begin{tabular}{|c|c|c|c|c|c|c|c|}
\hline \multirow{2}{*}{$m$} & \multirow{2}{*}{$n$} & \multicolumn{3}{|c|}{ Optimization } & \multicolumn{3}{|c|}{ Exhaustive search } \\
\cline { 3 - 8 } & & $f^{*}$ & $H_{\mathrm{r}}$ & $\alpha$ & $f^{*}$ & $H_{\mathrm{r}}$ & $\alpha$ \\
\hline 2 & 1 & 14.18 & 2.19 & 0.61 & 14.18 & 2.19 & 0.50 \\
\hline 3 & 2 & 14.55 & 2.28 & 0.67 & 14.55 & 2.28 & 0.67 \\
\hline 5 & 2 & 15.22 & 2.23 & 0.60 & 15.22 & 2.22 & 0.60 \\
\hline 10 & 5 & 15.30 & 2.23 & 0.60 & 15.30 & 2.23 & 0.60 \\
\hline 20 & 11 & 15.08 & 2.25 & 0.60 & 15.08 & 2.25 & 0.60 \\
\hline
\end{tabular}

dimples are shown in Table 8 . The highest load was 0.0665 , which was obtained for 2 dimples and $H_{\mathrm{r}}=1.90$.

Previous studies showed that for the mass-conserving cavitation case, the pressure can be increased by shifting dimples within the cell [14]. Therefore, optimization was also performed for dimples shifted by half of the cell (Tables 9 and 10). When shifting dimples this way, the number of dimples in $x$ direction can be described as $n_{h}=$ floor $((\alpha L-L c X / 2) / L c X)+1$. The length of the inlet dimple in $x$ direction is halved. For rectangular (elliptical) dimples the highest value of load equal to $0.0711(0.0584)$ was obtained for 10 (3) dimples, with $H_{\mathrm{r}}=1.69(1.97)$.

Another optimization was performed for rectangular dimples shifted to the inlet of the cell in the $x$ direction, as shown in Fig. 8. A comparison of the optimization results with exhaustive search is shown in Table 11. The highest value of load equal to 0.0711 was obtained for 20 dimples, with $H_{\mathrm{r}}=1.69$.

For all cases, optimal $A_{\mathrm{r}}$ and $\alpha$ were the same as in Example 1 and the exhaustive search was conducted over the range of $H_{\mathrm{r}}$ used before.

Obtained optimal film height and pressure for 20 elliptical half-shifted dimples are shown in Fig. 9.

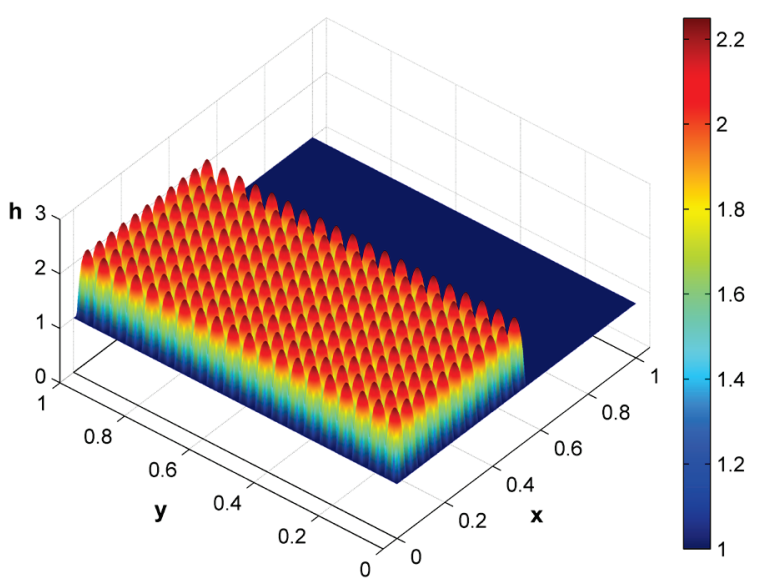

(a)

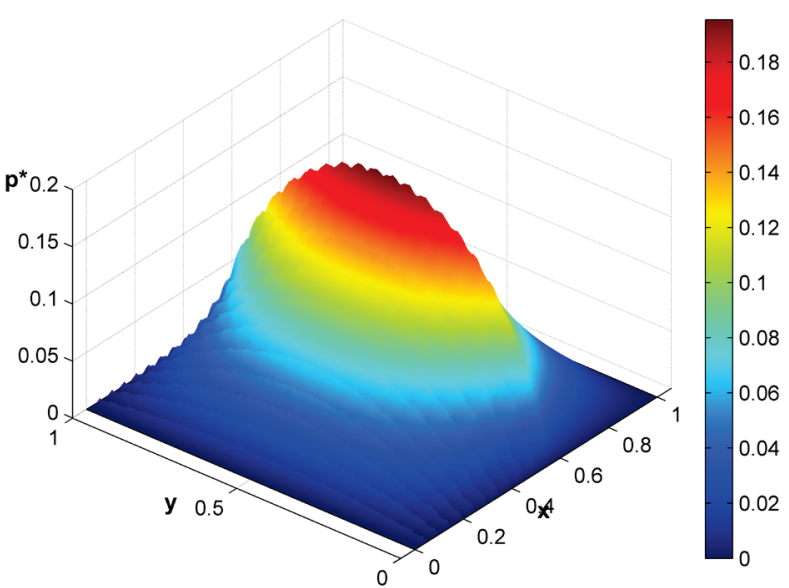

(b)

Fig. 7 Film height and pressure distribution with optimal friction coefficient for 20 elliptical dimples in y-direction 
Table 8 Optimal height ratio and dimensionless load for cell-centered rectangular dimples (mass-conserving cavitation)

\begin{tabular}{|c|c|c|c|c|c|}
\hline \multirow{2}{*}{$m$} & \multirow{2}{*}{$n$} & \multicolumn{2}{|c|}{ Optimization } & \multicolumn{2}{c|}{ Exhaustive search } \\
\cline { 3 - 6 } & & Load & $H_{\mathrm{r}}$ & Load & $H_{\mathrm{r}}$ \\
\hline 2 & 1 & 0.0665 & 1.90 & 0.0665 & 1.90 \\
\hline 3 & 1 & 0.0486 & 1.79 & 0.0486 & 1.80 \\
\hline 5 & 3 & 0.0514 & 1.77 & 0.0514 & 1.76 \\
\hline 10 & 6 & 0.0558 & 1.76 & 0.0558 & 1.75 \\
\hline 20 & 12 & 0.0577 & 1.76 & 0.0577 & 1.76 \\
\hline
\end{tabular}

Table 10 Optimal height ratio and dimensionless load for half shifted elliptical dimples (mass-conserving cavitation)

\begin{tabular}{|c|c|c|c|c|c|}
\hline \multirow{2}{*}{$m$} & \multirow{2}{*}{$n$} & \multicolumn{2}{|c|}{ Optimization } & \multicolumn{2}{c|}{ Exhaustive search } \\
\cline { 3 - 6 } & & Load & $H_{\mathrm{r}}$ & Load & $H_{\mathrm{r}}$ \\
\hline 2 & 1 & 0.0481 & 2.00 & 0.0481 & 2.00 \\
\hline 3 & 2 & 0.0584 & 1.97 & 0.0585 & 2.03 \\
\hline 5 & 3 & 0.0559 & 2.04 & 0.0559 & 2.04 \\
\hline 10 & 6 & 0.0551 & 2.06 & 0.0551 & 2.06 \\
\hline 20 & 11 & 0.0565 & 2.10 & 0.0565 & 2.10 \\
\hline
\end{tabular}

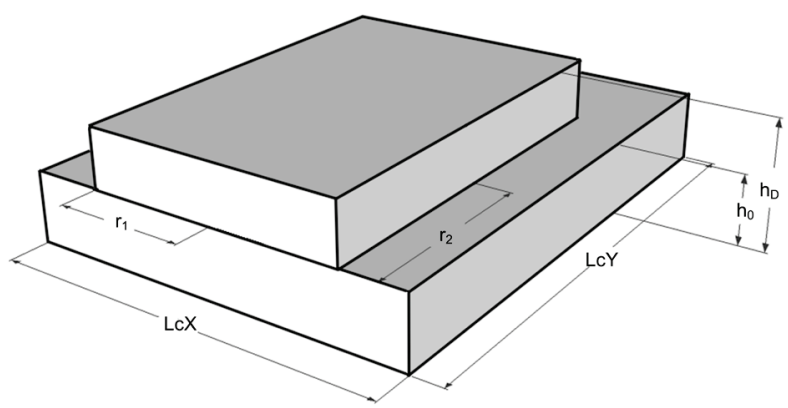

Fig. 8 Rectangular dimple cell with dimple shifted to the inlet

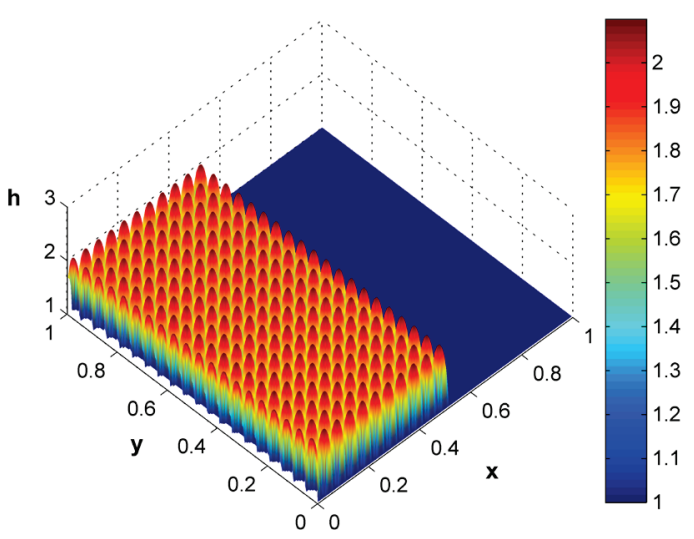

(a)

Table 9 Optimal height ratio and dimensionless load for half shifted rectangular dimples (mass-conserving cavitation)

\begin{tabular}{|c|c|c|c|c|c|}
\hline \multirow{2}{*}{$m$} & \multirow{2}{*}{$n$} & \multicolumn{2}{|c|}{ Optimization } & \multicolumn{2}{c|}{ Exhaustive search } \\
\cline { 3 - 6 } & & Load & $H_{\mathrm{r}}$ & Load & $H_{\mathrm{r}}$ \\
\hline 2 & 1 & 0.0507 & 1.64 & 0.0507 & 1.64 \\
\hline 3 & 1 & 0.0708 & 1.68 & 0.0708 & 1.69 \\
\hline 5 & 3 & 0.0708 & 1.68 & 0.0708 & 1.69 \\
\hline 10 & 6 & 0.0711 & 1.69 & 0.0711 & 1.69 \\
\hline 20 & 12 & 0.0708 & 1.69 & 0.0708 & 1.70 \\
\hline
\end{tabular}

Table 11 Optimal height ratio and dimensionless load for rectangular dimples shifted to the inlet of the cell (mass-conserving cavitation)

\begin{tabular}{|c|c|c|c|c|c|}
\hline \multirow{2}{*}{$m$} & \multirow{2}{*}{$n$} & \multicolumn{2}{|c|}{ Optimization } & \multicolumn{2}{c|}{ Exhaustive search } \\
\cline { 3 - 6 } & & Load & $H_{\mathrm{r}}$ & Load & $H_{\mathrm{r}}$ \\
\hline 2 & 1 & 0.0708 & 1.68 & 0.0708 & 1.68 \\
\hline 3 & 2 & 0.0614 & 1.66 & 0.0614 & 1.66 \\
\hline 5 & 3 & 0.0668 & 1.67 & 0.0668 & 1.67 \\
\hline 10 & 6 & 0.0708 & 1.68 & 0.0708 & 1.68 \\
\hline 20 & 11 & 0.0711 & 1.69 & 0.0711 & 1.69 \\
\hline
\end{tabular}


Example 4: Optimization of textured surface for the maximum load capacity, Reynolds equation considering mass-conserving cavitation and temperature change

Optimization of texture shapes was also performed for parallel slider bearing in the presence of temperature changes. For this optimization, the Reynolds equation was coupled with the energy equation. Since temperature changes are dimensional, the following Reynolds equation was used [27]:

$$
\begin{gathered}
\left(\frac{\partial^{2} p}{\partial x^{2}}+\frac{\partial^{2} p}{\partial y^{2}}\right) \int_{o}^{h} M \partial z+\left(\frac{\partial p}{\partial x} \frac{\partial}{\partial x}+\frac{\partial p}{\partial y} \frac{\partial}{\partial y}\right) \int_{o}^{h} M \partial z \\
+U \frac{\partial}{\partial x} \int_{o}^{h} N \partial z=0
\end{gathered}
$$

where

$$
\begin{gathered}
M=\int_{0}^{z} \frac{z}{\eta_{p}} d z-\frac{\int_{0}^{h} \frac{z}{\eta_{p}} d z \int_{0}^{z} \frac{d z}{\eta_{p}}}{\int_{0}^{h} \frac{d z}{\eta_{p}}} \\
M=\int_{0}^{z} \frac{z}{\eta_{p}} d z-\frac{\int_{0}^{h} \frac{z}{\eta_{p}} d z \int_{0}^{z} \frac{d z}{\eta_{p}}}{\int_{0}^{h} \frac{d z}{\eta_{p}}}
\end{gathered}
$$

\begin{tabular}{|c|c|}
\hline \multicolumn{2}{|l|}{ Bearing characteristics } \\
\hline Pad length & $0.1 \mathrm{~m}$ \\
\hline Pad width & $0.0667 \mathrm{~m}$ \\
\hline Minimum film height & $25 \mu \mathrm{m}$ \\
\hline Sliding velocity $U$ & $5 \mathrm{~m} / \mathrm{s}$ \\
\hline \multicolumn{2}{|l|}{ Lubricant properties } \\
\hline Viscosity at bearing inlet $\eta_{0}$ & $0.04 \mathrm{~Pa} \mathrm{~s}$ \\
\hline Temperature at bearing inlet $\mathrm{T}_{0}$ & $30{ }^{\circ} \mathrm{C}$ \\
\hline Density of the lubricant $\rho_{0}$ & $860 \mathrm{~kg} / \mathrm{m}^{3}$ \\
\hline Exponential viscosity-temperature coefficient & $0.03 \mathrm{~K}^{-1}$ \\
\hline Thermal conductivity of the lubricant $K$ & $0.15 \mathrm{~W} / \mathrm{mK}$ \\
\hline Specific heat of the lubricant $c_{p}$ & $1670 \mathrm{~J} / \mathrm{kgK}$ \\
\hline
\end{tabular}

The temperature change is governed by the energy equation:

Table 12 Bearing configuration

Table 13 Optimal height ratio and load for half shifted rectangular dimples (temperature-dependent viscosity and mass-conserving cavitation)

\begin{tabular}{|c|c|c|c|c|c|}
\hline \multirow{2}{*}{$m$} & \multirow{2}{*}{$n$} & \multicolumn{2}{|c|}{ Optimization } & \multicolumn{2}{c|}{ Exhaustive search } \\
\cline { 3 - 6 } & & Load [N] & $H_{\mathrm{r}}$ & Load [N] & $H_{\mathrm{r}}$ \\
\hline 2 & 2 & 7864 & 1.70 & 7865 & 1.71 \\
\hline 3 & 3 & 7001 & 1.69 & 7001 & 1.69 \\
\hline 5 & 5 & 7525 & 1.67 & 7525 & 1.68 \\
\hline 10 & 9 & 7817 & 1.70 & 7817 & 1.71 \\
\hline 20 & 18 & 8222 & 1.76 & 8222 & 1.76 \\
\hline
\end{tabular}

$$
\begin{aligned}
& \frac{\partial}{\partial x}\left(\rho u c_{p} T\right)+\frac{\partial}{\partial y}\left(\rho v c_{p} T\right)+\frac{\partial}{\partial z}\left(\rho w c_{p} T\right)-K \frac{\partial^{2} T}{\partial z^{2}} \\
& =\eta\left[\left(\frac{\partial u}{\partial z}\right)^{2}+\left(\frac{\partial v}{\partial z}\right)^{2}\right]
\end{aligned}
$$

Isothermal boundary condition [27] was chosen, i.e. bearing faces have fixed temperature and temperature changes are only within the lubricant.

The relation between viscosity and temperature is defined by:

$$
\eta_{p}=\eta_{0} e^{-\gamma T}
$$

The pad dimensions and bearing operating conditions are shown in Table 12.

The 2D Reynolds equation including mass-conserving cavitation from Example 3 was also used. To include temperature effect, the mean viscosity through the film thickness, in $z$ direction, was calculated. This allowed solving the mass-conserving Reynolds equation on a $2 \mathrm{D}$ grid. It was assumed that the error associated with this simplification was negligible due to small film thickness. Non-averaged (film height dependent) viscosity values were used for the calculation of velocities $u, v$ and $w$ in the $3 \mathrm{D}$ pressure-temperature equation.

Optimal results obtained for rectangular and elliptical dimples shifted by half of the cell in the $x$ direction are shown in Tables 13 and 14. The search was conducted over the range of $H_{\mathrm{r}}$ between 1.3 and 2.5 with steps of 0.01 . Optimal $A_{\mathrm{r}}$ and $\alpha$ found in this investigation were the same as in Example 3. The highest values of load equal to $8546 \mathrm{~N}$ and $8222 \mathrm{~N}$ were obtained for 2 elliptical and 20 rectangular dimples, respectively. Optimal film height ratios were 2.19 and 1.76 , respectively.

Obtained optimal film height and pressure for 10 elliptical half-shifted dimples are shown in Fig. 10.

\section{Discussion}

In this study, a uniform computational approach was developed to optimize surface texture parameters (height ratio, area density and textured portion of the slider length) in 2D hydrodynamic bearings governed by the Reynolds equation. The bearings satisfy the assumptions listed in Table 1. Using the approach developed optimal texture parameters that provide the maximum load/minimum friction coefficient were found for a $2 \mathrm{D}$

Table 14 Optimal height ratio and load for half shifted elliptical dimples (temperature-dependent viscosity and mass-conserving cavitation)

\begin{tabular}{|c|c|c|c|c|c|}
\hline \multirow{2}{*}{$m$} & \multirow{2}{*}{$n$} & \multicolumn{2}{|c|}{ Optimization } & \multicolumn{2}{c|}{ Exhaustive search } \\
\cline { 3 - 6 } & & Load [N] & $H_{\mathrm{r}}$ & Load [N] & $H_{\mathrm{r}}$ \\
\hline 2 & 2 & 8546 & 2.19 & 8546 & 2.20 \\
\hline 3 & 3 & 7029 & 2.11 & 7029 & 2.12 \\
\hline 5 & 4 & 7140 & 2.12 & 7140 & 2.12 \\
\hline 10 & 8 & 7094 & 2.14 & 7094 & 2.15 \\
\hline 20 & 17 & 7459 & 2.15 & 7459 & 2.15 \\
\hline
\end{tabular}




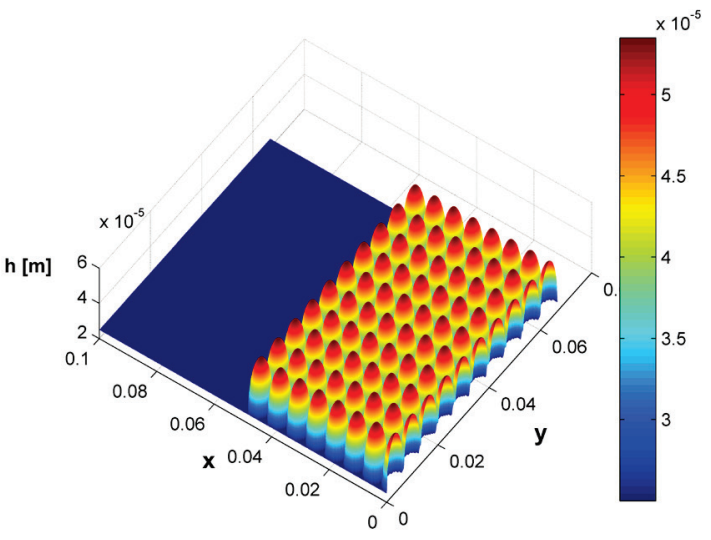

(a)

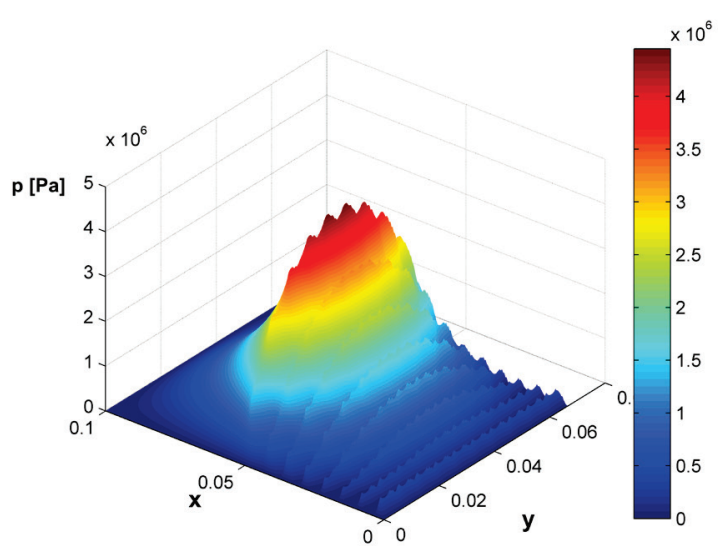

(b)

Fig. 10 Optimal film height and pressure distribution for 10 elliptical half shifted dimples, mass-conserving cavitation and viscosity decreasing with temperature

parallel bearing textured with rectangular and elliptical dimples. Additionally, the developed optimization approach was extended to account for mass-conserving cavitation and viscosity varying with temperature. The results of the optimization agree with the exhaustive search conducted and with the findings reported in literature $[6,12,23,33]$. This indicates that the approach developed is accurate and applicable to $2 \mathrm{D}$ bearings with parallel textured surfaces.

\subsection{Number of dimples $(m)$}

Optimal results depend on the number of dimples. In the case of rectangular cell-centered dimples, the two highest load capacities were obtained for 2 dimples regardless of cavitation and temperature changes. This is because the one row of dimples obtained resembles the optimal Rayleigh step bearing [4], and consequently the load capacity is high. With an increasing number of dimples, both in rows and columns, the load capacity decreases as a result of the textured surface having more "interdimple gaps" and thus being less similar to the step bearing. When the dimple number increases to 5 and above, the size of "interdimple gaps" decreases and thus the optimal step bearing is approximated better, giving a high load capacity. These findings agree with studies conducted by Dobrica and Fillon [14] who suggested that resemblance to the Rayleigh step (or a pocket slider) plays a significant role in generating hydrodynamic lift. This was also confirmed by Brizmer et al. [12] who concluded that lift was generated due to the collective effect of the dimples.

In the case of elliptical dimples, the influence of the dimple number on load capacity was similar. Load capacity increased significantly for more than 10 dimples in $y$-direction. This difference may be explained by the fact that the smooth, rounded shape of elliptical dimples is less similar to the optimal Rayleigh step shape as compared to the rectangular dimples.

\subsection{Position of dimple within cell}

Rectangular dimples shifted to the inlet of the cell and elliptical dimples shifted by a half of the cell generate significant hydrodynamic lifting force. The increase in the force can amount to $25 \%$ as compared to cell-centered dimples. This agrees with the results of Dobrica and Fillon who found that partial texturing with rectangular dimples placed at the inlet of the contact can give an increase of 50\% in lift [14]. This may suggest that hydrodynamic effect is more pronounced when film height at the bearing inlet is higher (as with shifted dimples). The differences between shifted by a half and fully shifted or cell-centered elliptical dimples may be explained by the fact that when the dimple half starts at the edge of the cell, the bearing resembles the optimal step more. A change in the number of rectangular dimples supporting optimal load was also observed. However, a small number of shifted rectangular dimples (between 10 and 20 dimples in y-direction) is required to achieve the highest load capacities.

\subsection{Height ratio $\left(H_{\mathrm{r}}\right)$}

In the load capacity optimization, optimal height ratios were ranging from 1.68 to 1.90 for rectangular dimples. These values agreed with results presented in [36], where the optimal ratio is approximately 1.7 for a parallel slider textured with 25 square dimples. They are also comparable to the optimal step bearing height ratio, which is 1.87 [27]. Obtained optimal height ratio for elliptical dimples was higher, ranging from 1.97 to 2.21. This agrees with results presented in [15] where the optimal experimentally obtained height ratio is 2.2 . Similarly, in [12] the optimal height ratio for elliptical dimples is equal to 2.25 . This suggests that dimple volume plays a role in generating hydrodynamic lift. In elliptical dimples, a larger height ratio is required to obtain the volume of the rectangular dimple that has the the same base area.

Optimal height ratio values were higher for dimples 
located in the cell centers and lower when they were shifted towards the inlet of the cell. This suggests that the shifting of dimples generates a significant lift increase, and then a high height ratio is not necessary. Optimal height ratios were also larger when the viscosity decreased with temperature. This is because a larger height ratio is needed to alleviate this negative effect.

\subsection{Area density $\left(A_{\mathrm{r}}\right)$ and textured portion of the slider} length $(\alpha)$

Irrespective of the dimple shape, cavitation algorithm applied and temperature effects considered, the optimal load and friction coefficient were always obtained for the maximum possible value of area density. For elliptical dimples, optimum area density was 0.78 , similar to $76 \%$ obtained from a numerical search in [15]. Also, in [37], the load was confirmed to increase with the dimple density. Maximum average pressure was obtained for dimple density of $65 \%$ and more. Experimental results shown in [15] also prove that maximum possible area density is optimal.

This finding agrees well with results previously published for parallel sliders textured with elliptical [12] and square [6] dimples, i.e. the texture density is high, individual dimples do not play a major role, and the bearing surface shape resembles the optimal step shape.

It was found that for both rectangular and elliptical dimples, approximately $60 \%$ of the slider length should be textured. This has support in a previous study [12], where the maximum load was obtained for a $65 \%$ textured parallel square slider (elliptical micro-dimples). Other studies showed that full surface texturing does not contribute to or has minimal effect on the generation of hydrodynamic lift in parallel sliders, while partial texturing can generate lift, as it brings the bearing closer to the optimal step/pocket $[14,38,39]$.

\subsection{Cavitation algorithm}

Optimal load and height ratio values depend on the cavitation algorithm used. The highest load values were obtained for the Reynolds boundary condition, followed by the half-Sommerfeld boundary condition and the mass-conserving cavitation algorithm. This agrees with results reported by Qiu and Khonsari [40], who observed that for a single dimple, both the Reynolds and half-Sommerfeld conditions overestimated the pressure generation and that the Reynolds condition predicted a load capacity larger than the half-Sommerfeld condition and the mass-conserving cavitation algorithm.

The half-Sommerfeld condition predicts film rupture in the diverging region of the bearing and simply replaces all negative pressures with 0 . Therefore, it implies discontinuity of flow between zero and non-zero pressure regions [27], and although simple to implement, it is unphysical and may lead to inaccurate results. The Reynolds condition correctly predicts film rupture, but does not consider film reformation. As a result, the Reynolds boundary condition may underestimate the cavitated area, thereby giving a falsely optimistic result, which was already stated by Ausas et al. [23] and confirmed in the current study. By contrast, in this study the half-Sommerfeld boundary condition gave results that, although still slightly overestimated, were close to those obtained with the mass-conserving cavitation algorithm. Therefore, we suggest that the half-Sommerfeld condition, due to its simplicity and ease of numerical application, should be chosen for the parallel sliders used in this study.

\subsection{Viscosity varying with temperature}

It was confirmed in this study that the decrease in viscosity due to the temperature rise can cause a drop in load carrying capacity of a bearing [41,42]. Thus, obtained dimple height ratios have to be higher to support load equivalent to that in the optimization without temperature change taken into consideration. The influence of textured portion of the slider length and dimple area density on the load capacity is the same as in our other examples.

Previous research showed that in the presence of viscosity decrease due to temperature rise, the load capacity is reduced and bearing surface shape has greater influence on the load capacity than when temperature change is not considered $[17,43]$. This confirms the importance of including temperature effects in optimization studies.

\subsection{Friction coefficient optimization}

For the friction coefficient, optimal height ratios for rectangular and elliptical dimples were in the range 1.76-1.81 and 2.19-2.28, respectively. The results obtained for rectangular dimples are consistent with a previous study of a parallel slider, where optimal ratios ranged from 1.7 to 1.9 [34]. The friction coefficient was researched for elliptical dimples in [44]. The maximum tested height ratio was 2.0 and a decrease in the coefficient value was observed with increasing height ratio. This agrees with our results where the initial height ratio equals 1.3. Optimal textured portion of slider length $\alpha$ decreased with the increasing number of dimples in $y$ direction. This is consistent with a study conducted by Pascovici et al. [34] in which the optimal lengths are within the interval $(0.6,0.8)$. Friction coefficient always decreased with the increasing texture density. This has support in studies of friction in sliders textured with elliptical [44] and rectangular [6, 34] dimples.

In the friction coefficient optimization, optimal values of the textured portion $\alpha$ obtained using the proposed approach and the exhaustive search were different in cases of 2, 5, 20 rectangular and 2 elliptical dimples (Tables 6 and 7). This can be explained by the fact that the exhaustive search has a large step size (0.01 as opposed to $10^{-8}$ in the optimization routine). We repeated the search using a small size of $10^{-5}$. Results obtained (i.e. $0.72,0.62$ and 0.60 for 2,5 and 20 rectangular and 0.58 for 2 elliptical dimples) were similar to the optimal values listed in Tables 6 and 7 . 
4.8. Limitations of the current study and future work

Conducted study has several limitations. The computations are based on the Reynolds equation and are therefore subject to simplifying assumptions as stated in Table 1.

No inertia was assumed in the lubricant flow. Nonetheless, fluid inertia can have significant effects on bearing performance. This was confirmed by Dobrica and Fillon [34], who determined a range of dimple sizes and Reynolds numbers for which inertia effects become significant in 1D textured sliders. In previous studies, inertia effects were included by using the Navier-Stokes equations [16,45]. However, since the approach presented herein bases on the Reynolds equation, the algorithm that could be used was presented by Arghir [46]. It allows an approximation the effects by including film discontinuities into the Reynolds equation.

It was assumed that viscosity of the lubricant does not change due to shear stress. This is satisfied only for Newtonian fluids. To perform the optimization for a bearing lubricated with a non-Newtonian fluid, it is necessary to use the appropriate rheological model, such as the power law, Bingham or Hershel-Bulkley model. The constitutive equation developed by Gecim for multigrade oils [47] or its modification made by Paranjpe [48] and the generalized Reynolds equation for three-dimensional slider bearings developed by El Khlifi [49] could be applied.

Incompressible flow between surfaces was assumed in this study. However, the approach developed can also be applied to bearings lubricated by compressible fluids. For example, the surface texture shape optimization can be performed for gas bearings governed by the Reynolds equation with a Burgdorfer's correction for thin air gaps [50] or the Reynolds equation for a hydrostatic compressible flow over a single dimple $[51,5]$.

In this work, the lubricant flow was laminar. Nonetheless, the flow can be turbulent (for Reynolds numbers greater than 1000), especially in high speed bearings. Turbulent flows can be described with an appropriate model, such as $k-\varepsilon$ [53] or Elrod, Ng and Pan [39] turbulence models. Consequently, the proposed approach could be extended to the Navier-Stokes equation solution in order to include turbulent flow effects.

In solving hydrodynamic bearing equation, the no-slip boundary condition is generally applied. This assumption was also made in current study. However, some recent research predicted slip in the flow of water between hydrophobic surfaces. Such surfaces are not yet in commercial use, however they may be applied in the future, so considering this prediction, a Reynolds equation incorporating slip/no-slip conditions was developed [54]. This equation can be applied for texture shape optimization considering slip conditions.

In this study, the Reynolds equation has been extended to account for mass-conserving cavitation and temperature-dependent viscosity. Nevertheless, the approach could be developed further, incorporating extended forms of the Reynolds equation, to take into account other phenomena that are discussed in [47-52,55-57].

The approach proposed has also some numerical limitations. The finite difference and finite volume codes were not optimized and computations for dense meshes are lengthy. Multigrid algorithm could be applied as a remedy.

\section{Conclusion}

From the work conducted the following conclusions can be drawn:

- An approach was developed that allows optimizing bearing surface textured with rectangular or elliptical dimples for 2D parallel sliders governed by the Reynolds equation using nonlinear programming techniques. Effects of cavitation and temperature change have been included.

- The approach provides optimal height ratio, area density of dimples and textured portion of the slider length.

- Although initial results were presented for bearings that satisfied the simplifying assumptions, given in Table 1, the approach can be modified (as already shown on some examples) in such a way that, e.g. inertia effects and slip/no-slip conditions can be included.

In the future, it is planned to develop an optimization technique for bearing textures in hydrodynamic contacts governed by $1 \mathrm{D}$ and 2D Navier-Stokes equations. The ultimate goal is to develop a unified computational approach for the optimization of 3D hydrodynamic and elastohydrodynamic lubrication contacts for any geometry of texture shapes and any lubricant rheology. The initial work conducted on 1D [7] and 2D bearings governed by the Reynolds equation is the first step towards this development.

\section{Acknowledgements}

The authors would like to thank the School of Mechanical and Chemical Engineering, University of Western Australia for its support during the writing of this paper. Agata Guzek wishes to thank the Commonwealth Government of Australia for the award of an IPRS scholarship and the University of Western Australia for the award of an UPAIS scholarship.

\section{Nomenclature}

$a$ - vector of textured surface parameters

$A_{\mathrm{r}}$ - area density of dimples:

$$
\boldsymbol{a}=\left[H_{r} A_{r} \alpha\right]
$$

for rectangular dimples: $A_{r}=\frac{4 r_{1} r_{2}}{L c X \cdot L c Y}$ 


$$
\text { for elliptical dimples: } \quad A_{r}=\frac{\pi r_{p}^{2}}{L c X \cdot L c Y}
$$

$B(L)$ - bearing width (length)

$c_{p}$ - fluid specific heat

$e$ - elliptical dimple elongation coefficient

$$
e=h_{0}\left(H_{r}-1\right) / r_{p}
$$

$g$ - objective function

$h^{*}$-dimensionless fluid film thickness $\quad h^{*}=h / h_{0}$

$h_{0}-$ minimum film thickness

$h_{\mathrm{D}}-$ dimple heigth

$H_{\mathrm{r}}$ - dimple height ratio

$H_{r}=h_{D} / h_{0}$

$f$ - friction coefficient

$$
f=\frac{F}{W}
$$

$F$ - friction force

$F^{*}$-dimensionless friction force

$K$ - fluid thermal conductivity

$L c X(L c Y)$ - dimple cell width (length)

$M, N$-auxiliary integrals in the temperature equation

$m$ - number of dimples in $y$-direction

$n_{h}$ - number of dimples in $x$-direction (for half-shifted dimples)

$$
n_{h}=\text { floor }((\alpha L-L c X / 2) / L c X)+1
$$

$r_{1}\left(r_{2}\right)$ - rectangular dimple half length in $x(y)$ direction

$r_{p}$ - radius of elliptical dimple base $\quad r_{p}=2 r_{1} \sqrt{A_{r} / \pi}$

$p$ - pressure

$p^{*}$-dimensionless pressure

$p_{0}-$ reference pressure

$p^{*}=p / p_{0}$

$T$ - temperature

$p_{0}=U \eta L / h_{0}^{2}$

$U$ - runner velocity

$W$ - load capacity

$u, v, w$ - velocity components

$x, y, z$-coordinates

$x^{*}, y^{*}$-dimensionless coordinates $\quad x^{*}=x / L$

$$
y^{*}=y / L
$$

$X_{r}, Y_{r}$ - relative coordinates inside dimple cell with respect to the centre of the cell

$$
\begin{gathered}
X_{r}=\bmod (x, L c X)-L c X / 2 \\
Y_{r}=\bmod (y, L c Y)-L c Y / 2
\end{gathered}
$$

$\alpha$ - textured portion of the slider length

$\eta$ - fluid viscosity

$\theta$ - fractional film content, $\theta=1$ in lubricated regions, $0<\theta<1$ in cavitated regions

$\gamma$ - exponent of viscosity-temperature dependence

$\eta_{p}-$ predicted dynamic viscosity

$\eta_{0}-$ dynamic viscosity at the reference temperature $T_{0}$

$\rho$ - fluid density

$\tau^{*}-$ dimensionless shear stress

\section{Nonlinear programming method}

$$
\tau^{*}=\frac{1}{h^{*}}+\frac{h^{*}}{2} \frac{d p^{*}}{d x}
$$

$g$ - objective function $\nabla g$ - gradient of objective function

$\boldsymbol{a}_{l b}$ - lower bound on parameter vector

$\boldsymbol{a}_{u b}$ - upper bound on parameter vector

$\boldsymbol{H}$ - Hessian matrix

$k$ - iteration number

${ }^{T}$ - matrix transpose

$\boldsymbol{d}$ - search direction

$\beta$ - step size

\section{References}

[1] Moore, D. F., "A History of Research on Surface Texture Effects," Wear, 13, 1969, 381-412.

[2] Costa, H. L. and Hutchings, I. M., "Hydrodynamic Lubrication of Textured Steel Surfaces under Reciprocating Sliding Conditions," Tribology International, 40, 2007, 1227-1238.

[3] Etsion, I., "State of the Art in Laser Surface Texturing," Trans. ASME, Journal of Tribology, 127, 1, 2005, 248-253.

[4] Rayleigh, O. M., "Notes on the Theory of Lubrication," Philosophical Magazine, 35, 1, 1918, $1-12$.

[5] Rahmani, R., Shirvani, A. and Shirvani, H., "Optimization of Partially Textured Parallel Thrust Bearings with Square-Shaped Micro-Dimples," Tribology Transactions, 50, 3, 2007, 401-406.

[6] Dobrica, M., Fillon, M., Pascovici, M. and Cicone, T., "Texturing Effects in Plane-Inclined Slider Bearings," Proceedings of ASME/STLE International Joint Tribology Conference IJTC2007, San Diego, California, USA, October 22-24, 2007, 269-271.

[7] Guzek, A., Podsiadlo, P. and Stachowiak, G. W., "A Unified Computational Approach to the Optimization of Surface Textures: One Dimensional Hydrodynamic Bearings," Tribology Online, 5, 3, 2010, 150-160.

[8] Kettleborough, C. F., "The Stepped Thrust Bearing - A Solution by Relaxation Methods," Trans. ASME, Journal of Applied Mechanics, 76, 1954, 19-24.

[9] Rohde, S. M. and McAllister, G. T., "On the Optimization of Fluid Film Bearings," Proceedings of the Royal Society of London Series A-Mathematical Physical and Engineering Sciences, 351, 1667, 1976, 481-497.

[10] McAllister, G. T. and Rohde, S. M., "An Optimization Problem in Hydrodynamic Lubrication Theory," Applied Mathematics \& Optimization, 2, 3, 1975, 223-235.

[11] Etsion, I., "Improving Tribological Performance of Mechanical Components by Laser Surface Texturing," Tribology Letters, 17, 4, 2004, 733-737.

[12] Brizmer, V., Kligerman, Y. and Etsion, I., "A Laser Surface Textured Parallel Thrust Bearing," Tribology Transactions, 46, 3, 2003, 397-403. 
[13] Etsion, I., "Experimental Investigation of Laser Surface Textured Parallel Thrust Bearings," Tribology Letters, 17, 2, 2004, 295-300.

[14] Dobrica, M. B., Fillon, M., Pascovici, M. D. and Cicone, T., "Optimizing Surface Texture for Hydrodynamic Lubricated Contacts Using a Mass-Conserving Numerical Approach," Proceedings of the Institute of Mechanical Engineers Part J: Journal of Engineering Tribology 224, Special Issue, 2010, 737-750.

[15] Ma, C. and Zhu, H., “An Optimum Design Model for Textured Surface with Elliptical-Shape Dimples under Hydrodynamic Lubrication," Tribology International, 44, 9, 2011, 987-995.

[16] Cupillard, S., Glavatskih, S. and Cervantes, M. J., "Computational Fluid Dynamics Analysis of a Journal Bearing with Surface Texturing," Proceedings of the Institution of Mechanical Engineers Part J: Journal of Engineering Tribology, 222, 2, 2008, 97-107.

[17] Cupillard, S., Glavatskih, S. and Cervantes, M. J., "D Thermohydrodynamic Analysis of a Textured Slider," Tribology International, 42, 2009, 1487-1495.

[18] Wang, N., "Engineering Optimum Design of Fluid-Film Lubricated Bearings," Tribology Transactions, 43, 3, 2000, 377-386.

[19] van Ostayen, R. A. J., van Beek, A. and Munnig-Schmidt, R., "Film Height Optimization of Hydrodynamic Slider Bearings," Proceedings of ASME/STLE International Joint Tribology Conference IJTC2007, San Diego, California, USA, October 22-24, 2007, 1-4.

[20] van Ostayen, R. A. J., "Film Height Optimization of Dynamically Loaded Hydrodynamic Slider Bearings," Tribology International, 43, 10, 2010, 1786-1793.

[21] Buscaglia, G. C., Ausas, R. F. and Jai, M., "Optimization Tools in the Analysis of Micro-Textured Lubricated Devices," Inverse Problems in Science and Engineering, 14, 4, 2006, 365-378.

[22] Reeves, C. R. and Rowe, J. E., "Genetic Algorithms: Principles and Perspectives: a Guide to GA Theory," Kluwer Academic Publishers, Norwell, MA, USA, 2002.

[23] Ausas, R. F., Ragot, P., Leiva, J., Jai, M., Bayada, G. and Buscaglia, G. C., "The Impact of the Cavitation Model in the Analysis of Microtextured Lubricated Journal Bearings," Trans. ASME, Journal of Tribology, 129, 2007, 868-875.

[24] Folberg, L. and Jakobsson, B., "The Finite Journal Bearing, Considering Vaporization," Trans. Chalmers Univ. Tech, 190, Goteborg, 1957, 1-116.

[25] Elrod, H. G., "A Cavitation Algorithm," Trans. ASME, Journal of Lubrication Technology, 103, 3, $1981,350-354$.
[26] Elrod, H. G. and Adams, M., "A Computer Program for Cavitation and Starvation Problems," Proceedings of the First Leeds-Lyon Symposium on Cavitation and Related Phenomena in Lubrication, Leeds, UK, September 1974, 37-41.

[27] Stachowiak, G. W. and Batchelor, A., "Engineering Tribology," Elsevier-Butterworth-Heineman, Woburn, MA, USA, 2005.

[28] Tala-Ighil, N., Fillon, M. and Maspeyrot, P., "Effect of Textured Area on the Performances of a Hydrodynamic Journal Bearing," Tribology International, 44, 3, 2011, 211-219.

[29] Etsion, I. and Halperin, G., "A Laser Surface Textured Hydrostatic Mechanical Seal," ASLE Transactions, 45, 3, 2002, 430-434.

[30] Dobrica, M. B. and Fillon, M., "About the Validity of Reynolds Equation and Inertia Effects in Textured Sliders of Infinite Width," Proceedings of the Institute of Mechanical Engineers Part J: Journal of Engineering Tribology 223, 2009, 69-78.

[31] Nocedal, J. and Wright, S. J., "Numerical Optimization," Springer, New York, NY, USA, 2006.

[32] COMSOL Multiphysics 3.5a, User Manual, COMSOL Inc., 2009.

[33] Tala-Ighil, N., Maspeyrot, P., Fillon, M. and Bounif, A., "Effects of Surface Texture on Journal-Bearing Characteristics under Steady-State Operating Conditions," Proceedings of the Institution of Mechanical Engineers Part J: Journal of Engineering Tribology, 221, 6, 2007, 623-633.

[34] Pascovici, M. D., Cicone, T., Fillon, M. and Dobrica, M. B., "Analytical Investigation of a Partially Textured Parallel Slider," Proceedings of the Institution of Mechanical Engineers, Part J: Journal of Engineering Tribology, 223, 2, 2009, 151-158.

[35] Rahmani, R., Shirvani, A. and Shirvani, H., "Analytical Analysis and Optimisation of the Rayleigh Step Slider Bearing," Tribology International, 42, 5, 2009, 666-647.

[36] Pascovici, M. D., Marian, V. and Gaman, D., "Analytical and Numerical Approach of Load Carrying Capacity for Partially Textured Slider," Proceedings of International Nanotribology Conference Nano Sikkim II: Friction and Biotribology, Peeling, Sikkim, India, November 8-12, 2004, 1-10.

[37] Qiu, Y. and Khonsari, M. M., "Performance Analysis of Full-Film Textured Surfaces with Consideration of Roughness Effects," Trans. ASME, Journal of Tribology, 133, 2, 2011, 021704-021710.

[38] Tønder, K., "Dimpled Pivoted Plane Bearings: Modified Coefficients," Tribology International, 43, 12, 2010, 2303-2307. 
[39] Brunetiere, N. and Tournerie, B., "Study of Hydrostatic Mechanical Face Seals Operating in a Turbulent Rough Flow Regime," Trans. ASME, Journal of Tribology, 131, 3, 2009, 032202.

[40] Qiu, Y. and Khonsari, M. M., "On the Prediction of Cavitation in Dimples Using a Mass-Conservative Algorithm," Trans. ASME: Journal of Tribology, 131, 3, 2009, 041702.

[41] Tichy, J. A. and Smith, R. N., "An Analytical Solution for Thermal Behavior of the Step Thrust Bearing," Trans. ASME, Journal of Lubrication Technology, 102, 1, 1980, 34-40.

[42] Yu, T. H. and Sadeghi, F., "Thermal Effects in Thrust Washer Lubrication," Trans. ASME, Journal of Tribology, 124, 1, 2002, 166-177.

[43] Dobrica, M. B. and Fillon, M., "Thermohydrodynamic Behavior of a Slider Pocket Bearing," Trans. ASME, Journal of Tribology 128, 2006, 312-318.

[44] Han, J., Fang, L., Sun, J. and Ge, S., "Hydrodynamic Lubrication of Microdimple Textured Surface Using Three-Dimensional CFD," Tribology Transactions, 53, 6, 2010, 860-870.

[45] Sahlin, F., Glavatskih, S. B., Almqvist, T. and Larsson, L., "Two-Dimensional CFD-Analysis of Micro-Patterned Surfaces in Hydrodynamic Lubrication," Trans. ASME, Journal of Tribology, 127, 1, 2005, 96-102.

[46] Arghir, M., Alsayed, A. and Nicolas, D., "The Finite Volume Solution of the Reynolds Equation of Lubrication with Film Discontinuities," International Journal of Mechanical Sciences, 44, 10, 2002, 2119-2132.

[47] Gecim, B., "Non-Newtonian Effects of Multigrade Oils on Journal Bearing Performance," Tribology Transactions, 33, 3, 1990, 384-394.

[48] Paranjpe, R. S., "Analysis of Non-Newtonian Effects in Dynamically Loaded Finite Journal Bearings Including Mass Conserving Cavitation," Trans. ASME, Journal of Tribology, 114, 4, 1992, 736-744.
[49] Khlifi, M. E., Souchet, D., Hajjam, M. and Bouyahia, F., "Numerical Modeling of Non-Newtonian Fluids in Slider Bearings and Channel Thermohydrodynamic Flow," Trans. ASME, Journal of Tribology, 129, 3, 2007, 695-699.

[50] Jai, M., Buscaglia, G. C. and Iodanoff, I., "Multi-Constrained Optimization of Running Characteristics of Mechanisms Lubricated with Compressible Fluid," Trans. ASME, Journal of Tribology, 126, 2004, 132-136.

[51] van Odyck, D. E. A. and Venner, C. H., "Compressible Stokes Flow in Thin Films," Trans. ASME, Journal of Tribology, 125, 2003, 543-551.

[52] Feldman, Y., Kligerman, Y. and Etsion, I., "The Validity of the Reynolds Equation in Modeling Hydrostatic Efects in Gas Lubricated Textured Parallel Surfaces," Trans. ASME, Journal of Tribology, 128, 2006, 345-350.

[53] Luan, Z. and Khonsari, M. M., "Computational Fluid Dynamics Analysis of Turbulent Flow within a Mechanical Seal Chamber," Trans. ASME, Journal of Tribology, 129, 1, 2007, 120-128.

[54] Li, Y. and Menon, A. K., "The Development and Implementation of Discrete Texture for the Improvement of Tribological Performance," Trans. ASME, Journal of Tribology, 117, 2, 1995, 279-284.

[55] Arghir, M., "Theoretical Analysis of the Incompressible Laminar Flow in a Macro-Roughness Cell," Trans. ASME, Journal of Tribology, 125, 2, 2003, 309-318.

[56] Salant, R. F. and Fortier, A. E., "Numerical Analysis of a Slider Bearing with a Heterogeneous Slip/No-Slip Surface," Tribology Transactions, 47, 2004, 328-334.

[57] Christensen, H., "Some Aspects of the Functional Influence of Surface Roughness in Lubrication," Wear, 17, 1971, 149-162. 